\title{
Inhibitors of the Renal Outer Medullary Potassium Channel
}

\section{Benjamin Blass*}

Temple University School of Pharmacy, 3307 North Broad Street, Philadelphia, Pennsylvania 19140, United States

Title:

Patent Application Number:

Priority Application:

Inventors:

\section{Assignee Company:}

Disease Area:

Summary:

Important Compound Classes:

Definitions:

$\mathrm{Z}$ is

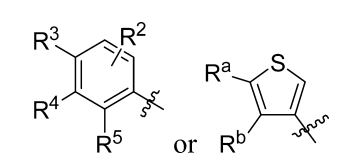

$\mathrm{R}^{1}$ is

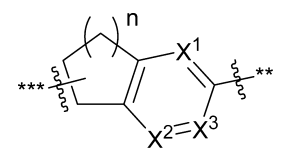

wherein ${ }^{* * *}$ indicates attachment to the carbonyl carbon and ${ }^{* *}$ indicates attachment to the tetrazolyl ring;

$\mathrm{X}$ is $\mathrm{O}, \mathrm{NH}$, or $\mathrm{S}$;

$m$ is an integer selected from 1 or 2 ;

$n$ is an integer selected from 1 or 2 ;

$\mathrm{X}^{1}, \mathrm{X}^{2}$, and $\mathrm{X}^{3}$ are each independently selected from $\mathrm{C}\left(\mathrm{R}^{7}\right)$ or $\mathrm{N}$, provided that at least one of $\mathrm{X}^{1}, \mathrm{X}^{2}$, and $\mathrm{X}^{3}$ must be $\mathrm{N}$ and at most two of $\mathrm{X}^{1}, \mathrm{X}^{2}$, and $\mathrm{X}^{3}$ are $\mathrm{N}$;

$\mathrm{R}^{\mathrm{a}}$ is $\mathrm{CN}$;

$\mathrm{R}^{\mathrm{b}}$ is $\mathrm{H}$ or $\mathrm{C}_{1-6}$ alkyl;

$\mathrm{R}^{2}$ is $\mathrm{H}, \mathrm{F}, \mathrm{Cl}, \mathrm{C}_{1-6}$ alkyl, $\mathrm{C}_{3-6}$ cycloalkyl, or $\mathrm{OC}_{1-6}$ alkyl;

$\mathrm{R}^{3}$ is $\mathrm{H}, \mathrm{F}, \mathrm{Cl}, \mathrm{CN}, \mathrm{C}_{1-6}$ alkyl, $\mathrm{C}_{3-6}$ cycloalkyl, or $\mathrm{OC}_{1-6}$ alkyl;

$\mathrm{R}^{4}$ is $\mathrm{F}, \mathrm{Cl}, \mathrm{CN}, \mathrm{C}_{3-6}$ cycloalkyl, $\mathrm{OC}_{1-4}$ alkyl, or $\mathrm{N}$-tetrazolyl;

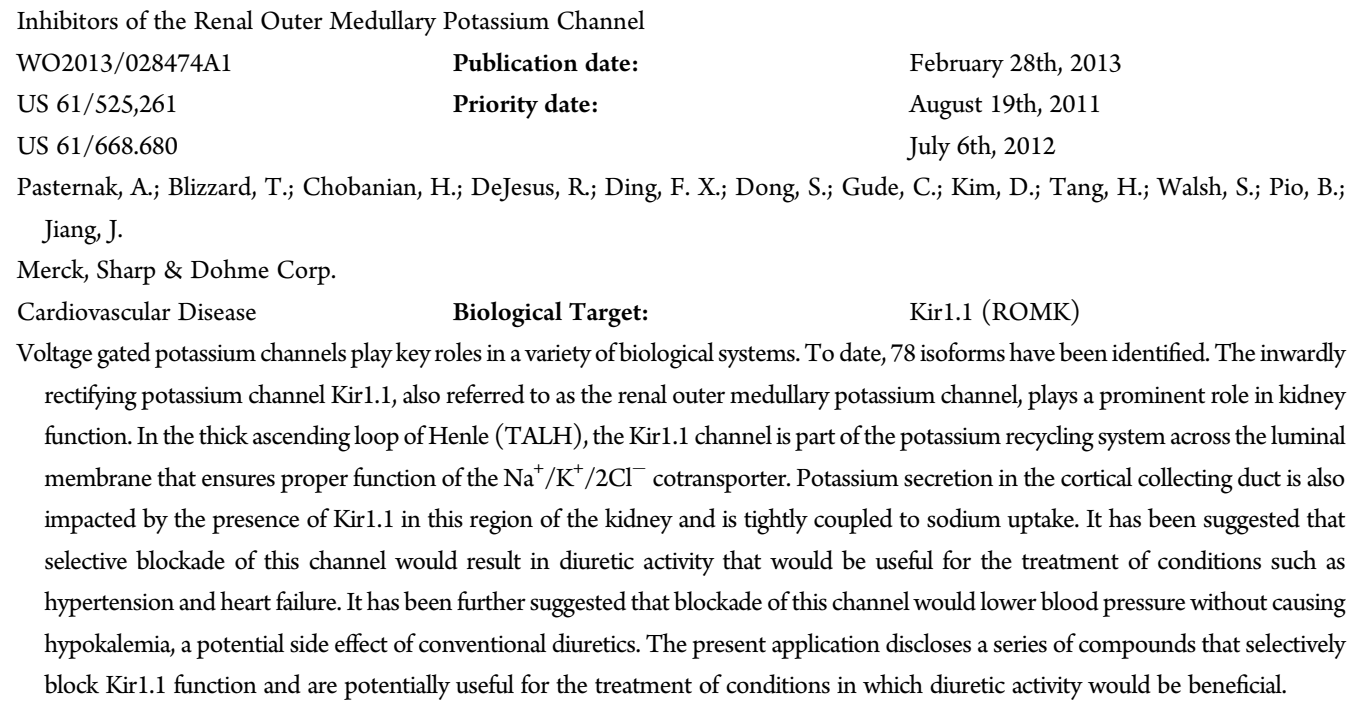

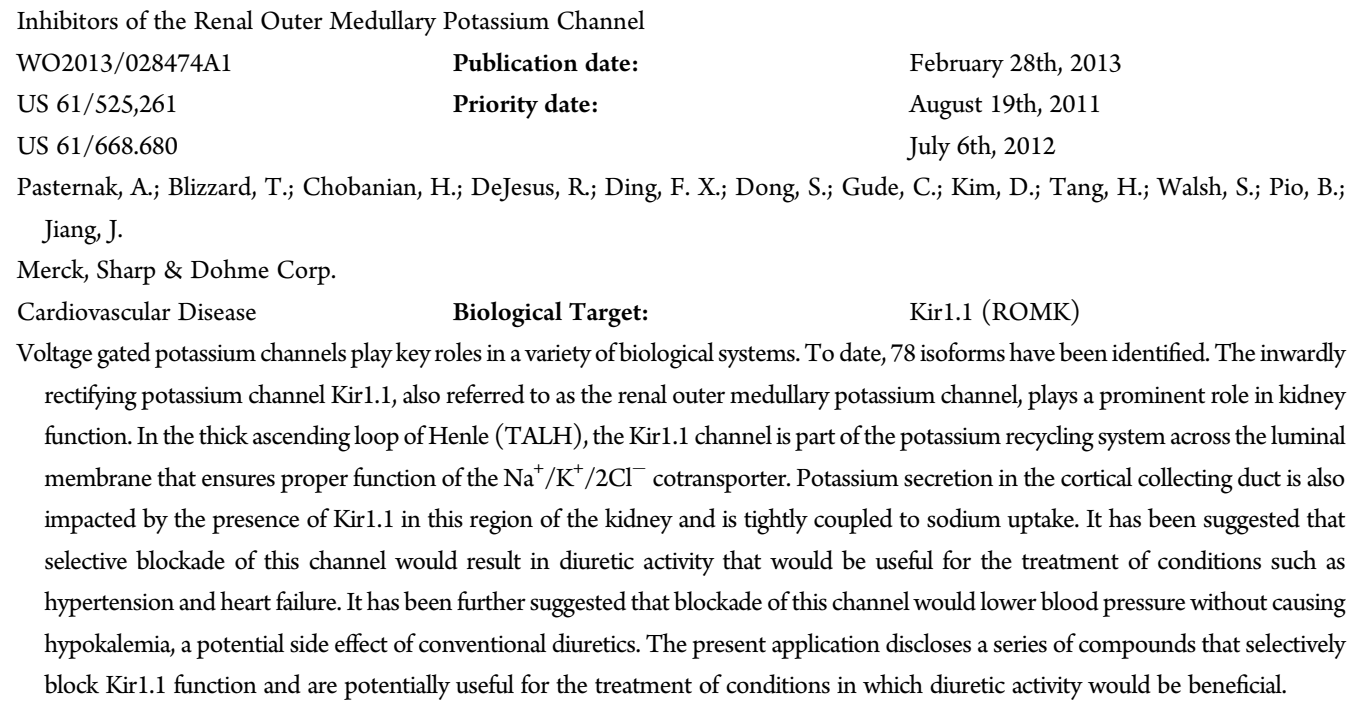

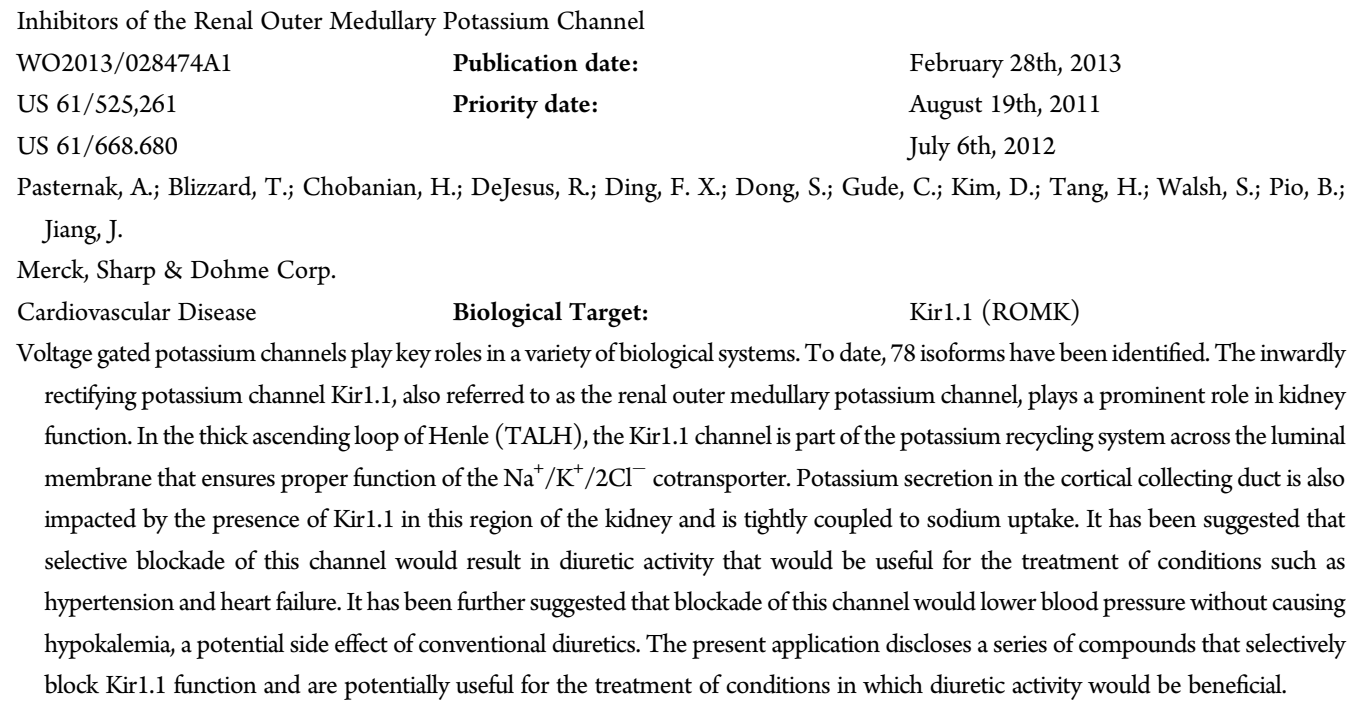

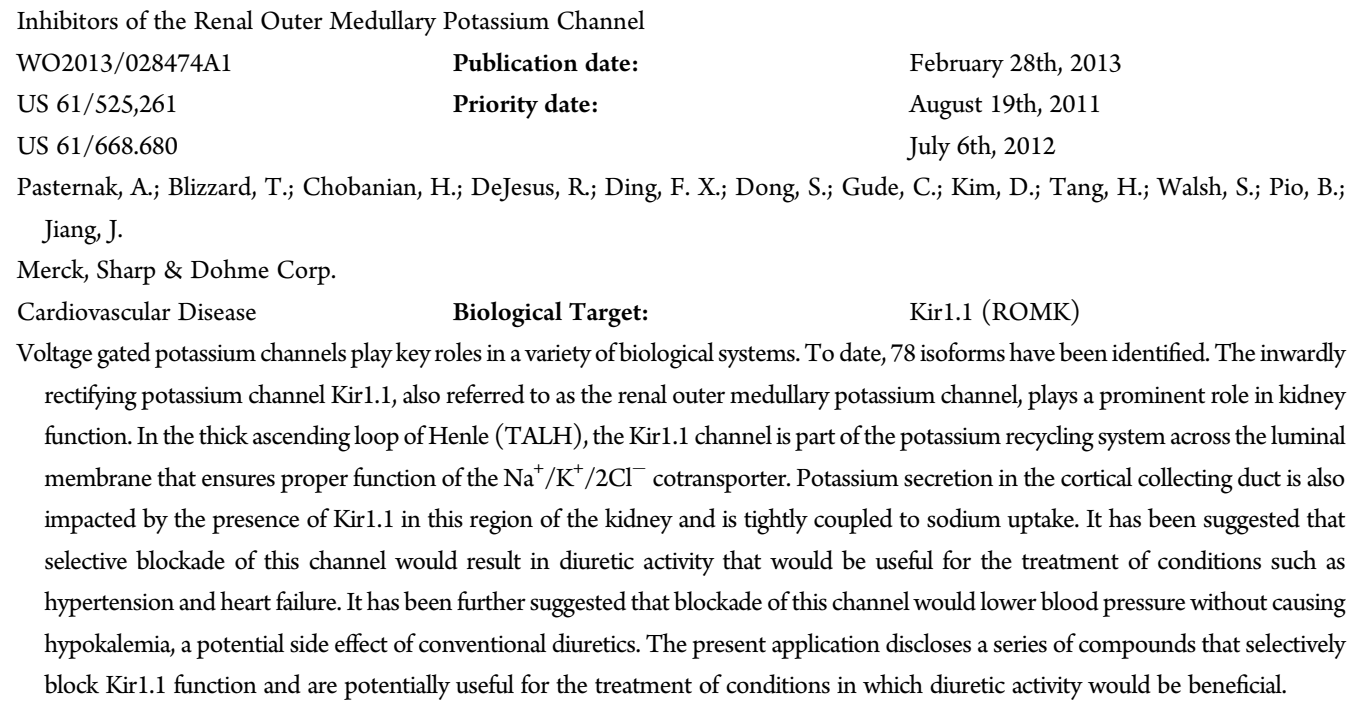

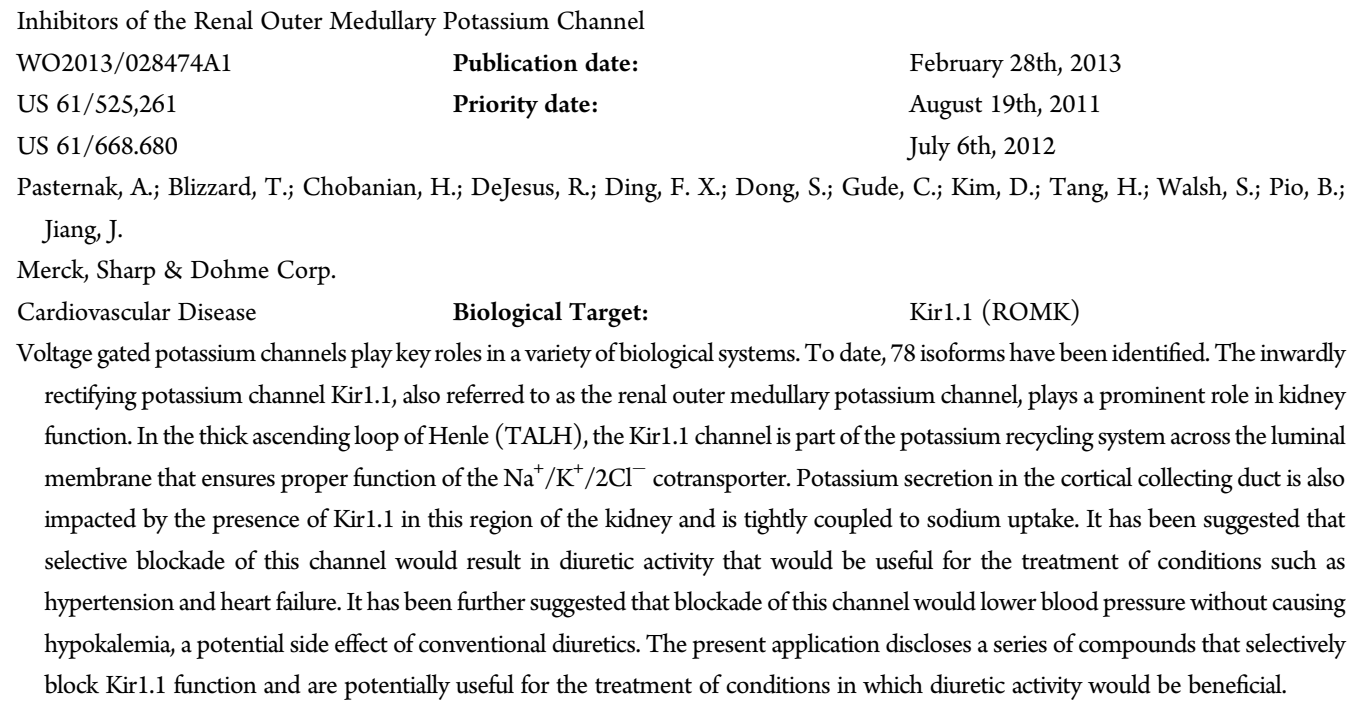

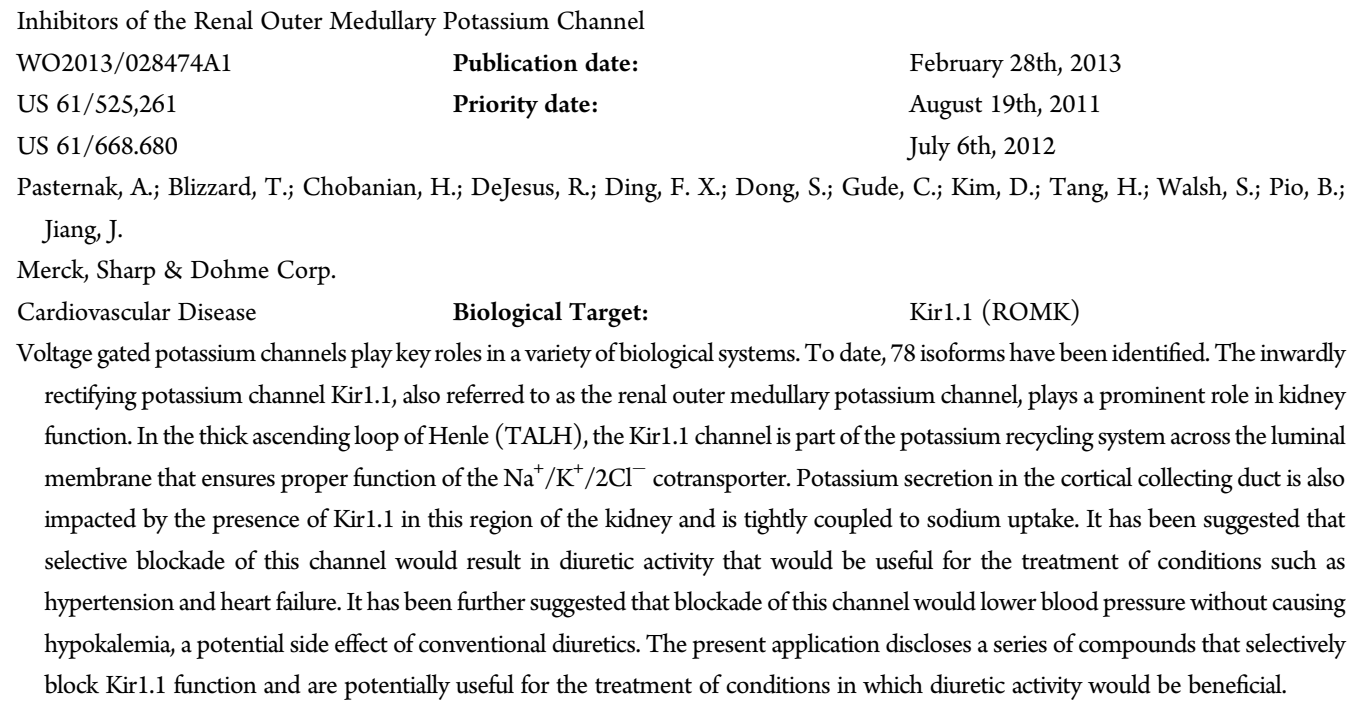

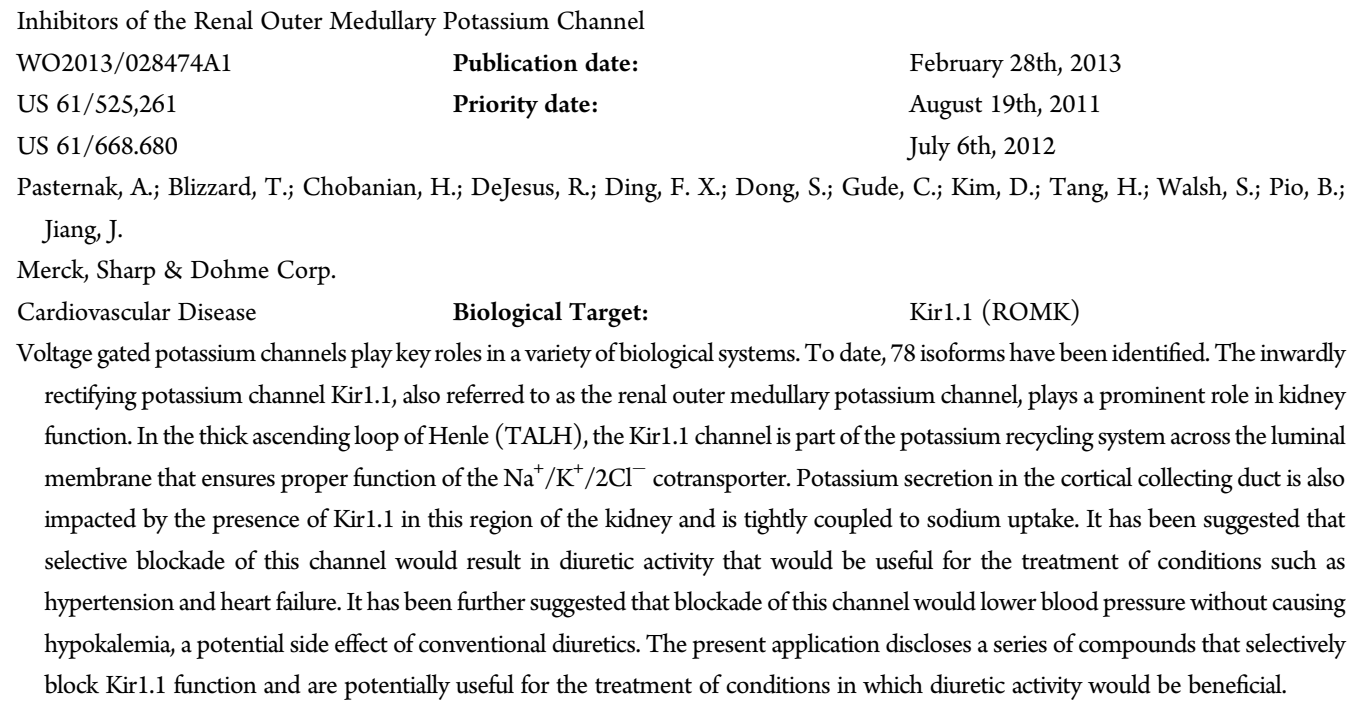

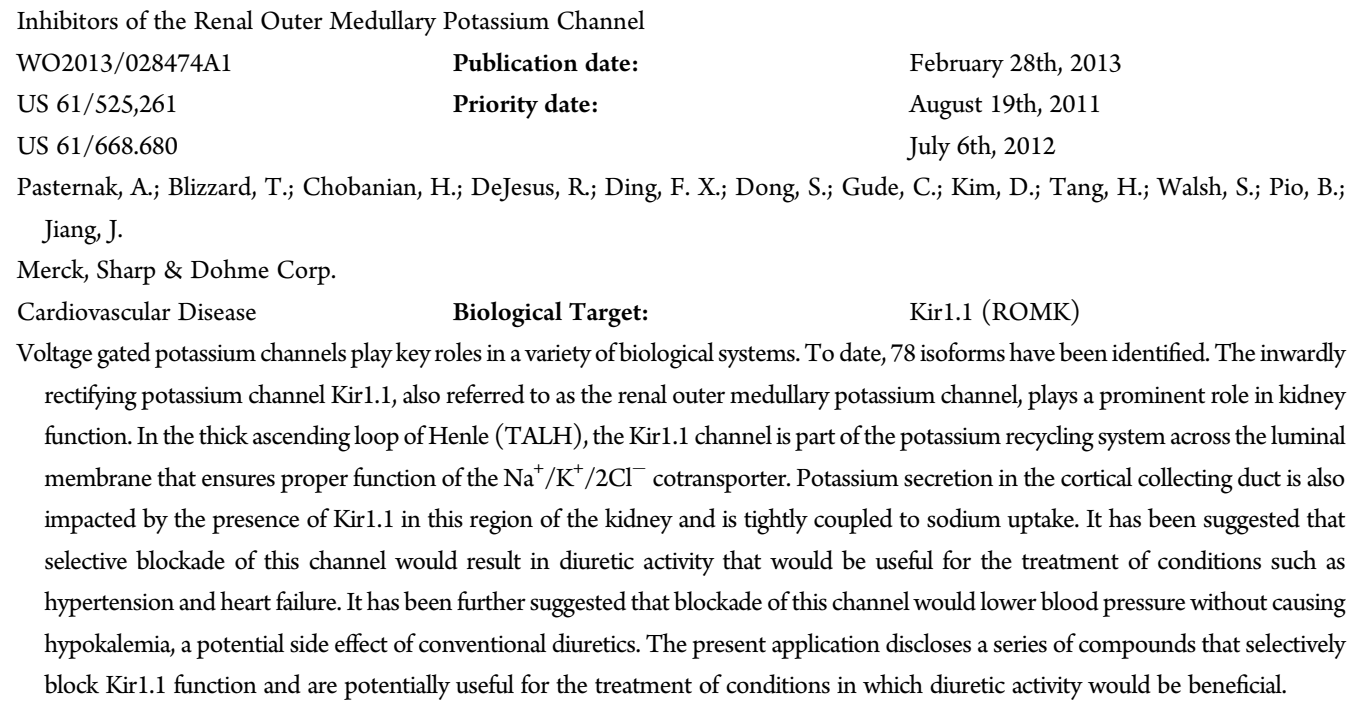

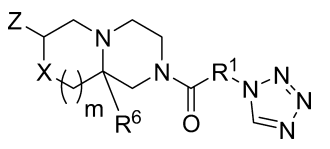


or $\mathrm{R}^{3}$ and $\mathrm{R}^{4}$ are joined together with the carbon atoms in the phenyl ring to which they are attached to form

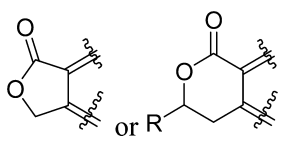

wherein $\mathrm{R}$ is $\mathrm{H}$ or $\mathrm{C}_{1-4}$ alkyl;

$\mathrm{R}^{5}$ is $\mathrm{H}, \mathrm{Cl}, \mathrm{F}, \mathrm{CN}, \mathrm{C}_{1-4}$ alkyl, $\mathrm{C}_{3-6}$ cycloalkyl, or $\mathrm{OC}_{1-4}$ alkyl;

Provided that, when $\mathrm{R}^{3}$ and $\mathrm{R}^{4}$ are not joined together, one and only one of $\mathrm{R}^{3}, \mathrm{R}^{4}$, or $\mathrm{R}^{5}$ is $\mathrm{CN}$;

$\mathrm{R}^{6}$ is $\mathrm{H}$ or $\mathrm{C}_{1-4}$ alkyl; and

$\mathrm{R}^{7}$ is $\mathrm{H}, \mathrm{F}, \mathrm{Cl}$, or $\mathrm{C}_{1-4}$ alkyl.

Key Structures:

Recent Review Articles:

Biological Assay:

Biological Data:<smiles>Cc1c([C@H]2CN3CCN(C(=O)C4CCc5nc(-n6cnnn6)ccc54)C[C@H]3CO2)ccc(F)c1C#N</smiles><smiles>O=C1OCc2cc([C@H]3CN4CCN(C(=O)C5CCc6nc(-n7cnnn7)ccc65)C[C@H]4CO3)ccc21</smiles><smiles>Cc1c([C@H]2CN3CCN(C(=O)C4CCc5nc(-n6cnnn6)ccc54)C[C@H]3CO2)csc1C#N</smiles><smiles>N#Cc1c(F)ccc([C@H]2CN3CCN(C(=O)C4CCc5nc(-n6cnnn6)ncc54)C[C@H]3CO2)c1Cl</smiles><smiles>N#Cc1c(F)ccc([C@H]2CO[C@H]3CN(C(=O)C4CCc5cc(-n6cnnn6)ncc54)CCN3C2)c1F</smiles><smiles>CC1=C2C=CC(F)=C(C(C#N)=C1)C2CN1CCN(C(=O)C2CCc3nc(-n4cnnn4)ccc32)CC1C[O]</smiles><smiles></smiles>

1. Welling, P. A.; Ho, K. A Comprehensive Guide to the ROMK Potassium Channel: Form and Function in Health and Disease. Am. J. Physiol. 2009, 297 (4), F849-F863.

2. Wang, W. H. Regulation of ROMK (Kir1.1) Channels: New Mechanisms and Aspects. Am. J. Physiol. Renal Physiol. 2006, 290 (1), F14-F19.

$\mathrm{K}_{\mathrm{ir}} 1.1$ Thallium flux assay, HEK293 cells stably expressing $\mathrm{hK}_{\mathrm{ir}} 1.1$, FluxOR, FLIRP Tetra 384. $\mathrm{K}_{\mathrm{ir}} 1.1$ whole cell voltage clamp, $\mathrm{CHO}$ cells stably expressing $\mathrm{hK}_{\mathrm{ir}} 1.1$, IonWorks Quattro.

\begin{tabular}{|c|c|c|c|c|c|}
\hline Entry & $\begin{array}{c}\mathrm{K}_{\text {ir }} 1.1 \mathrm{Th} \\
\text { Flux }(\mathrm{uM})\end{array}$ & $\begin{array}{c}\mathrm{K}_{\text {ir }} 1.1 \text { Patch } \\
\text { clamp }(\mathrm{uM})\end{array}$ & Entry & $\begin{array}{c}\mathrm{K}_{\text {ir } 1.1 \mathrm{Th}} \\
\text { Flux }(\mathrm{uM})\end{array}$ & $\begin{array}{c}\mathrm{K}_{\text {ir }} 1.1 \text { Patch } \\
\text { clamp (uM) }\end{array}$ \\
\hline 7 & 0.14 & 0.08 & 57 & 0.11 & 0.09 \\
\hline 15 & 0.54 & 0.06 & 81 & 0.17 & 0.06 \\
\hline 22 & 0.16 & 0.04 & 97 & 0.16 & 0.09 \\
\hline 55 & 0.14 & 0.06 & 103 & 0.18 & 0.10 \\
\hline
\end{tabular}

Claims:

16 Total claims.

13 Composition of matter claims.

3 Method of use claims.

\section{AUTHOR INFORMATION}

\section{Corresponding Author}

*Tel: 215-707-1085. E-mail: benjamin.blass@temple.edu.

Notes

The authors declare no competing financial interest. 